\title{
Potencial dendroclimático de Pinus pinceana Gordon en la Sierra Madre Oriental
}

\section{Dendroclimatic potential of Pinus pinceana Gordon in the Sierra Madre Oriental}

\author{
Miriam Santillán-Hernández¹, Eladio H. Cornejo-Oviedo², \\ José Villanueva-Díaz ${ }^{3}$, Julián Cerano-Paredes ${ }^{3}$, \\ Salvador Valencia-Manzo2 y Miguel Ángel Capó-Arteaga2
}

\begin{abstract}
RESUMEN
Se determinó la sensibilidad climática de Pinus pinceana Gordon y su potencial para reconstrucciones climáticas en diez de sus poblaciones localizadas en la región noreste (Zacatecas y Coahuila), norte-centro (San Luis Potosí) y centro (Hidalgo y Querétaro) de México. Se fecharon al año de formación de sus crecimientos anuales las poblaciones del noreste, centronorte y centro de la república; excepto la población de El Arenalito en la cual no se encontró un patrón similar de crecimiento. En las poblaciones del centro (MAG, TEP y CRI) se encontraron los árboles más jóvenes y como resultado se generaron cronologías de corta extensión; en contraste, en los sitios PAL y NOR se encontraron los árboles de mayor edad. Los valores de correlación $(0,61$ a 0,77$)$, sensibilidad media $(0,49$ a 0,69$)$ y relación señal-ruido $(11,72$ a 33,64$)$, fueron altos para las series dendrocronológicas generadas. Los parámetros estadísticos indican que Pinus pinceana es climáticamente sensible, además muestra un comportamiento similar a otras coníferas del norte de México empleadas en análisis dendrocronológicos previos. Con base en el análisis de función de respuesta (programa ARSTAN), se determinó que las poblaciones del noreste y centro-norte responden de manera significativa al periodo común de precipitación estacional enero-julio (estaciones invierno-primavera). Las poblaciones del centro no mostraron una respuesta significativa a la precipitación. La identificación de anillos con crecimiento reducido fue clave para encontrar patrones de crecimiento y realizar el fechado cruzado, básico para la generación de índices dendrocronológicos.
\end{abstract}

PALABRAS CLAVE:

Análisis de función de respuesta, anillos de árboles, dendroclimatología, sensibilidad climática media.

\begin{abstract}
The climate sensitivity of ten populations of Pinus pinceana Gordon was determined to analyze their potential for climate reconstruction purposes. The $P$. pinceana populations are distributed along a latitudinal gradient of the Sierra Madre Oriental and for this study are considered as northeast region (Zacatecas, Coahuila), north-central region (San Luis Potosi), and central region (Hidalgo, Queretaro). All populations were dated to the year of the annual growth formation except a population called "El
\end{abstract}

Programa Docente de Postgrado en Ciencias Forestales. Departamento Forestal. Universidad Autónoma Agraria Antonio Narro. Buenavista, Saltillo, Coahuila. edafo96@hotmail.com.

2 Universidad Autónoma Agraria Antonio Narro. Buenavista, Saltillo, Coahuila. cor61@prodigy.net.mx, svalencia@uaaan.mx,macapo@uaaan.mx.

3 Laboratorio de Dendrocronología. Instituto Nacional de Investigaciones Forestales, Agrícolas y Pecuarias. CENIDRASPA. Gómez Palacio, Durango. villanueva.jose@inifap.gob.mx, cerano.julian@inifap.gob.mx. 


\begin{abstract}
Arenalito" in the central region. The youngest trees were found at populations in the central region (MAG, TEP, and $\mathrm{CRI}$ ); therefore short tree-ring chronologies were developed for these sites. In contrast, for the northeastern region the populations PAL and NOR had the oldest trees and longer chronologies were developed for these sites. Intercorrelation values $(0,61$ to 0,77$)$, mean sensitivity $(0,49$ to 0,69$)$, and sign-to noise ratio values $(11,72$ to 33,64$)$ are considered high for dendrochronological purposes. The statistic parameters derived from the ARSTAN software indicated that $P$. pinceana is climate sensitive and shows a similar behavior to other conifer species currently used for dendroclimatic studies in northern Mexico. Response function analysis indicated that the populations located in the northeast and north-central regions significantly responded to the seasonal January-June precipitation (winter-spring seasons), but the central populations did not show a clear response. Defining patterns of reduced annual growth are basic to date this species.
\end{abstract}

\section{KEYWORDS:}

Response function analysis, tree rings, dendroclimatology, mean climatic sensitivity.

\section{INTRODUCCIÓN}

Los dendrocronólogos se refieren al término sensibilidad como la capacidad que tienen los árboles de una región determinada para reflejar la variabilidad climática a través de las fluctuaciones en el grosor de sus anillos de un año al siguiente. Esta condición puede ser observada en series tomadas de un conjunto de árboles que habitan una misma área y que presentan los mismos patrones de crecimiento en la variación de sus anillos; cuando esto sucede, se dice entonces que los árboles responden al mismo factor climático; es decir, son climáticamente sensibles (Fritts, 1976).

Los registros de los anillos de los árboles de coníferas sensitivas al clima constituyen una fuente de alta resolución aproximada y pueden ser usados para extender registros de la precipitación y de otras variables climáticas más allá de los registros históricos a los que se tiene acceso, a través de la generación de series de tiempo dendrocronológicas (Fritts, 1976).

En México existe una gran diversidad biológica de especies sensibles con potencial dendrocronólogico. A pesar de ello, se ha generado poca investigación con relación a los anillos de crecimiento (Villanueva et al., 2000), no obstante, que en la región noreste del país existen especies sensibles con alto potencial que son importantes para conocer la variabilidad climática en esta región (GonzálezElizondo et al., 2005).

En la actualidad, los estudios dendrocronológicos han tenido un surgimiento en México debido a la importancia científica que reviste el conocimiento del cambio climático global, impacto de patrones de circulación atmosférica y su efecto en el ciclo hidrológico, y en la disponibilidad actual y futura de los recursos hídricos, de alto impacto en actividades productivas y para consumo humano en el norte de México (Villanueva et al., 2007).

Las cronologías de anillos de árboles son particularmente importantes en nuestro país debido a que la mayoría de ellas están bien correlacionadas con la precipitación invernal y con los índices de El Niño/Oscilación del Sur (ENSO, por sus siglas en inglés) (Stahle et al., 2000). La influencia climática de ENSO se refleja en cronologías derivadas de coníferas del norte y noreste de México (Villanueva et al., 2004). Las reconstrucciones climáticas pueden ayudar a definir el impacto histórico de este fenómeno en la región y ayudar a estimar la probabilidad de sequías extremas en el futuro (Pohl et al., 2003). 
La mayoría de los estudios dendrocronológicos en el noreste de México se han desarrollado principalmente con los géneros Pinus y Pseudotsuga, por lo que en este estudio se analiza el potencial de Pinus pinceana para determinar su sensibilidad climática y la factibilidad para desarrollar reconstrucciones dendroclimáticas.

Pinus pinceana es una especie de pino piñonero que constituye una comunidad natural importante en la región semiárida de la Sierra Madre Oriental de México, donde cohabita con otros piñoneros. La especie se distribuye en los estados de Nuevo León, Coahuila, Zacatecas, San Luis Potosí, Hidalgo y Querétaro.

Pinus pinceana es una especie endémica considerada como rara por la NOM-ECOL-0592001 (Semarnat, 2004), y estudios dendrocronológicos pueden contribuir a desarrollar acciones para su conservación.

\section{OBJETIVO}

El objetivo de este estudio fue evaluar el potencial de reconstrucción climática de Pinus pinceana comparando las estadísticas básicas y la relación clima-crecimiento en un gradiente latitudinal de su distribución natural.

\section{METODOLOGÍA}

Las poblaciones estudiadas de Pinus pinceana se localizan en el centro y norte de la Sierra Madre Oriental; en el estado de Hidalgo se consideraron los rodales de San Cristóbal y El Arenalito; en Querétaro, El Tepozán y Maguey Verde; en Zacatecas, San José Carbonerillas y Cañón de las Bocas; en San Luis Potosí, Matehualilla y La Trinidad y en el estado de Coahuila, Palmas Altas y Norias (Figura 1).
Los sitios se muestrearon en el periodo de abril a diciembre de 2005; utilizando el muestreo selectivo, es decir, se seleccionaron individuos localizados en laderas con alta insolación, de apariencia longeva (Villanueva et al., 2005). Se extrajeron de uno a tres núcleos de crecimiento por árbol distribuidos en la periferia del fuste principal tratando de obtener la muestra lo más cercana al centro del árbol a una altura de 1,30 m empleando el taladro de Pressler. Las muestras se montaron en molduras de madera para facilitar su manejo, se pulieron con lijas de número 120 a 1200 para una mejor diferenciación de los anillos de crecimiento. El fechado de las series de crecimiento se llevó a cabo a través de técnicas dendrocronológicas estándar (Stokes y Smiley, 1968; Holmes, 1983), en el laboratorio de dendrocronología del INIFAP CENIDRASPA en Gómez Palacio, Durango. Fechadas las muestras, cada crecimiento individual se midió con un sistema Velmex a una resolución de 0,001 mm. La calidad del fechado y medición se verificó con el programa COFECHA (Holmes, 1983; Grissino-Mayer, 2001).

Generadas las bases de datos de ancho de anillo, a cada una de las muestras se les removieron las tendencias biológicas como edad, competencia y liberación no relacionadas con clima, utilizando el programa ARSTAN, al ajustar diferentes curvas (exponencial negativa, lineal, etc.) según el crecimiento de cada uno de los árboles y luego dividir cada valor anual de medición entre el valor obtenido de la curva. Esto generó series de tiempo estandarizadas (Cook, 1987).

El programa ARSTAN generó tres cronologías denominadas: Estándar, Residual y Arstan. Para los análisis se empleó la cronología residual, normalmente utilizada para reconstrucciones climáticas, ya que mediante el proceso de su generación se elimina la autocorrelación (Fritts, 1976). 


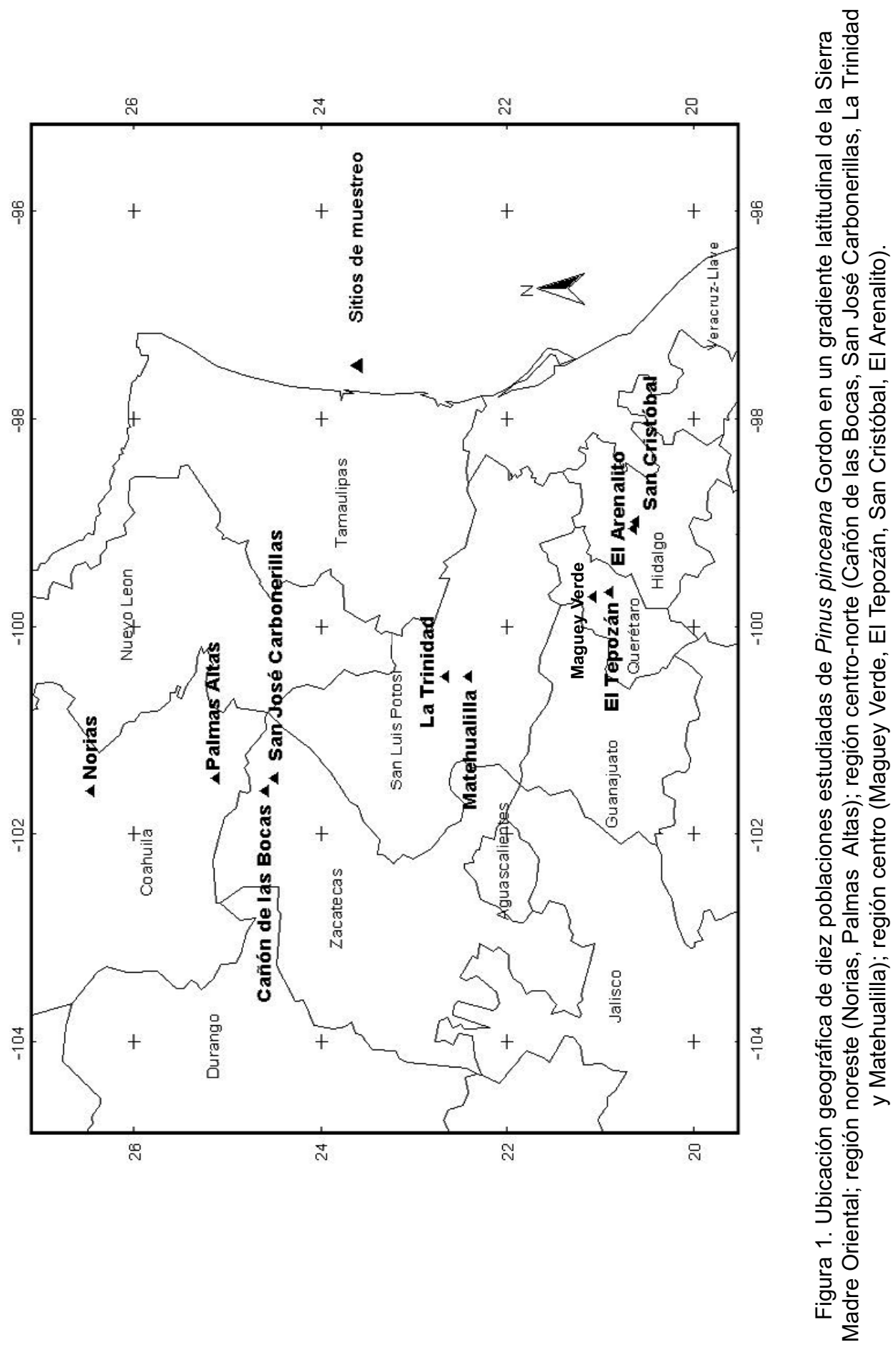


En función de los estadísticos de sensibilidad, correlación entre series, relación señal-ruido, autocorrelación de primer orden y desviación estándar generados por el programa ARSTAN, se determinó el potencial dendrocronológico de la especie para las diferentes poblaciones.

Para conocer la variabilidad común entre las diferentes poblaciones de Pinus pinceana se corrió un análisis de componentes principales mediante el uso del programa Statistica Kernel versión 5.5 (Stat Soft Inc., 2000); las cronologías de las diferentes poblaciones se agruparon en dos componentes, las poblaciones del norte que comprenden los estados de Coahuila, Zacatecas y San Luis Potosí presentan una misma variabilidad común (CP1), de igual manera las cronologías del centro que comprenden los estados de Hidalgo y Querétaro presentaron una misma variabilidad común (CP2). Dado lo anterior, se optó por trabajar con cronologías regionales por estado tanto para el norte como para el centro del país.

La respuesta climática o potencial de la especie para registrar la variabilidad de la precipitación se determinó mediante una función de respuesta, al correlacionar 49 años de datos instrumentales regionales que comprenden el periodo de 1950 a 1998, con los índices regionales de anillo generados para Coahuila, Zaca- tecas y San Luis Potosí. Los datos instrumentales de las estaciones meteorológicas se extrajeron de la base de datos ERIC II; las estaciones utilizadas fueron, dos localizadas en Coahuila (Sabinas y Viesca) y dos de San Luis Potosí (San Luis Potosí y Manantial de Media Luna) para la parte norte (Tabla 1). Para la parte centro, cronologías regionales de Hidalgo y Querétaro, se correlacionaron con datos climáticos de la estación Peñamiller, Querétaro, con 41 años de registro que comprenden el periodo de 1961 a 2001 (Tabla 1). Las estaciones climáticas se seleccionaron de acuerdo con su cercanía a los sitios de muestreo, la calidad de los datos registrados y su correlación con la cronología.

Finalmente, la relación se verificó con el programa PRECON que comprende un análisis de regresión múltiple, corriendo primeramente un "stepwise multiple regression" para la obtención de los componentes principales de las variables climáticas y finalmente un "bootstrap multiple regression" sobre los componentes para la estimación de los intervalos de confianza. En el análisis se consideraron 14 meses de precipitación, que se extiende de julio del año previo a agosto del año actual de crecimiento; el cual se realiza con la finalidad de observar la influencia en el crecimiento de las condiciones climáticas de meses del

Tabla 1. Estaciones climáticas cuyos registros fueron empleados para correr la función de respuesta con las cronologías regionales.

\begin{tabular}{lllccc}
\hline Regiones Estados & Estaciones climáticas & Longitud (O) & Latitud (N) & Periodo de datos \\
\hline \multirow{2}{*}{ Norte } & \multirow{2}{*}{ Coahuila } & Sabinas & $-101,283$ & 27,867 & $1941-2000$ \\
& \multirow{2}{*}{ San Luis Potosí } & Viesca & $-102,800$ & 25,350 & $1940-1973$ \\
& S. L. P. & $-100,967$ & 22,150 & $1949-2002$ \\
& Manantial de Media Luna & $-100,017$ & 21,850 & $1979-2002$ \\
\multirow{2}{*}{ Centro } & Querétaro & Peñamiller & $-99,814$ & 21,055 & $1961-2001$ \\
\hline
\end{tabular}


año anterior en el crecimiento del año en curso, procedimiento estadísticamente más confiable que un simple análisis de correlación (Fritts, 1990).

\section{RESULTADOS}

\section{Problemas de fechado con Pinus pinceana}

El fechado de la población ARE no fue posible, debido a que no se encontró un patrón definido en su crecimiento. En algunos sitios uno de los principales problemas encontrados para el fechado fue el porcentaje de anillos ausentes. En este aspecto, los sitios de la región centro-norte tuvieron los más altos porcentajes de anillos perdidos con $3,68 \%$ y $3,53 \%$ para las poblaciones TRI y MAT, respectivamente; ambas pertenecientes al estado de San Luis Potosí. La población NOR en Coahuila mostró el menor valor $(0,43 \%)$ de anillos ausentes (Tabla 2).
Las poblaciones que presentaron problemas de anillos falsos fueron ARE con $12 \%$, CRI con $10 \%$ y TEP con $2 \%$; situación que dificultó la generación de los índices dendrocronológicos para estos sitios (Tabla 2).

Las poblaciones CAN, CAR, MAT y TRI presentaron gran cantidad de microanillos, aunque no se realizaron conteos específicos para determinar el porcentaje que estos representan.

Con la excepción de dos de los sitios de la región centro, El Tepozán en el estado de Querétaro y San Cristóbal en el estado de Hidalgo, cuyas series generadas fueron de 57 y 49 años respectivamente, el resto de las cronologías se extiende en el tiempo por más de 100 años, destacando los sitios de Palmas Altas en Coahuila y San José Carbonerillas en Zacatecas como las series más longevas, 205 y 157 años, respectivamente.

Tabla 2. Problemas detectados en el fechado de las series de crecimiento de diez poblaciones de Pinus pinceana Gordon en su rango de distribución natural.

\begin{tabular}{|c|c|c|c|c|c|c|}
\hline Región & Estado & Población & $\begin{array}{c}\text { Total de } \\
\text { muestras fechadas }\end{array}$ & $\begin{array}{l}\text { Longitud de } \\
\text { las series }\end{array}$ & $\begin{array}{c}\text { Anillos } \\
\text { ausentes (\%) }\end{array}$ & $\begin{array}{c}\text { Anillos } \\
\text { falsos (\%) }\end{array}$ \\
\hline \multirow[t]{2}{*}{ Noreste } & Coahuila & NOR & 43 & 106 & 0,43 & 0 \\
\hline & & PAL & 25 & 205 & 2,26 & 0 \\
\hline \multirow[t]{4}{*}{ Centro-norte } & Zacatecas & CAN & 46 & 125 & 1,27 & 0 \\
\hline & & CAR & 43 & 157 & 2,93 & 0 \\
\hline & San Luis Potosí & MAT & 33 & 125 & 3,53 & 0 \\
\hline & & TRI & 49 & 131 & 3,68 & 0 \\
\hline \multirow[t]{4}{*}{ Centro } & Querétaro & MAG & 16 & 135 & 2,68 & 0 \\
\hline & & TEP & 6 & 57 & 0,00 & 2 \\
\hline & Hidalgo & CRI & 14 & 49 & 0,00 & 10 \\
\hline & & ARE & 0 & 0 & 0,00 & 12 \\
\hline
\end{tabular}


De igual manera, es importante señalar que en estos sitios existe el potencial para incrementar la extensión de las cronologías, enfocándose a la búsqueda de arbolado longevo, toma de muestras de tocones y madera sobre el piso forestal o semienterrada.

\section{Potencial dendrocronológico de Pinus pinceana}

Se determinó el potencial dendrocronológico de Pinus pinceana mediante el análisis de series de crecimiento, logrando analizar el mayor número de series en la región centro-norte. En las poblaciones TRI y CAN se fecharon 49 y 46 series, respectivamente. La menor cantidad de series se analizó para la región centro en TEP y CRI con 6 y 14 series, respectivamente (Tabla 3 ).

Los estadísticos derivados del análisis de ARSTAN que definen la calidad de la información climática obtenida de las cronologías de Pinus pinceana son la correlación, sensibilidad media, desviación estándar, relación señal-ruido y autocorrelación de primer orden (Holmes, 1983; Cook, 1987).

Los valores más altos de correlación se encontraron para el sitio PAL en la región noreste con un valor 0,77 . En la región centro-norte se encontraron valores de 0,74 y 0,69 en CAN, MAT y TRI, respectivamente. En el centro se registraron valores de 0,75 y 0,63 para $C R I$ y TEP, en ese orden (Tabla 3 ).

Las poblaciones de la región centronorte mostraron valores de 0,69, de 0,64 y 0,61 para los sitios MAT, TRI y CAR, respectivamente; los sitios NOR (para el noreste) y TEP (para el centro) indicaron valores de 0,53 y 0,51 , respectivamente (Tabla 3).
La desviación estándar fue mayor en la parte centro del rango de distribución de Pinus pinceana con un valor de 0,47 para CRI y de 0,46 para PAL. La población NOR ubicada en el noreste indicó un valor de 0,32 (Tabla 3).

La autocorrelación indicó similitud entre las series de crecimiento; de esta manera se obtuvieron valores de 0,20 a 0,29 para las poblaciones TRI y CAR ubicadas en el centro-norte y noroeste. Este parámetro fue muy variable para la parte centro, con valores de 0,09 a 0,39 en TEP y CRI, respectivamente (Tabla 3 ).

La especie registró valores de señalruido de 34,63 a 33,64 para los sitios CAR y CAN. Este parámetro decreció para los sitios MAG y TEP con valores de 1,35 y 11,72 respectivamente (Tabla 3 ).

\section{Función de respuesta}

En la región noreste se encontró que los meses de diciembre, febrero, mayo y julio respondieron de manera significativa $(p<0,05)$ al crecimiento de la especie, mientras que los meses de enero, marzo, abril y agosto influyen de manera positiva aunque no significativa $(p>0,05)$ (Figura 2a).

En la región centro-norte los meses que influyeron de manera significativa $(p<0,05)$ fueron diciembre del año previo al crecimiento y enero, febrero, marzo, mayo y julio del año actual de crecimiento; abril influye de manera positiva pero no significativa $(p>0,05)$ en el crecimiento (Figuras $2 \mathrm{~b}$ y $2 \mathrm{c}$ ).

El análisis de función de respuesta para las poblaciones del noreste y centronorte muestra una misma respuesta significativa $(p<0,05)$ para los meses de diciembre del año previo y febrero, marzo, mayo, julio y agosto del año actual de crecimiento; los meses de enero, abril 


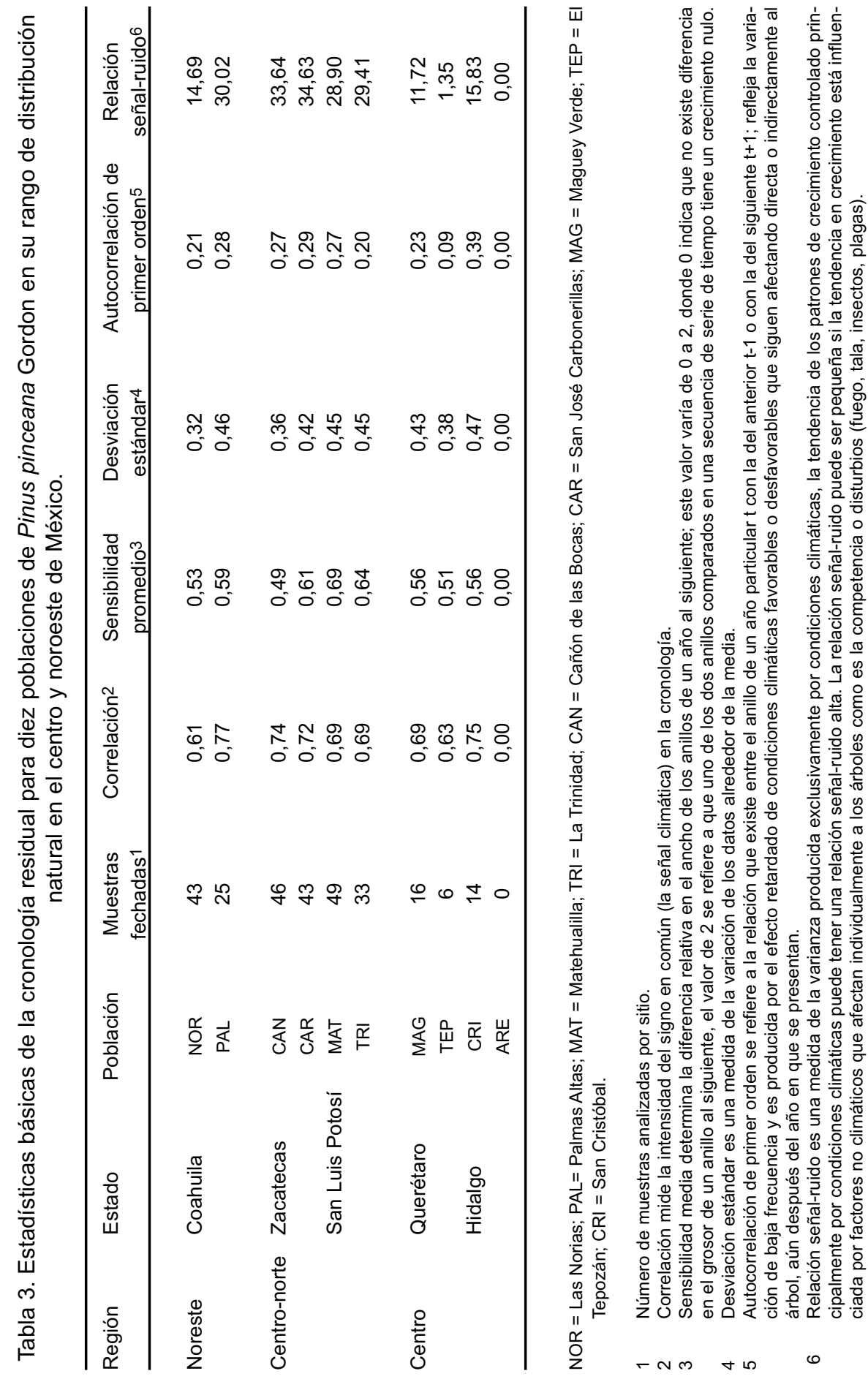


y junio muestran una respuesta no significativa $(p>0,05)$. Sin embargo, al realizar un análisis por periodos, se encontró que ambas regiones muestran una respuesta significativa $(p<0,05)$ para el periodo de precipitación estacional enero-julio, lo que indica que el crecimiento de la especie en esta parte de la República Mexicana está influenciado por el periodo de precipitación invierno-primavera (Figura 2).

La respuesta de la especie en la región centro no presentó un periodo de Iluvia común que defina su crecimiento; el análisis de función de respuesta para la población de Maguey Verde, Querétaro, mostró que los meses de enero, mayo y agosto se relacionan de manera significativa $(p<0,05)$ con el crecimiento; los meses de marzo y abril presentan una influencia positiva, aunque no significativa $(p>0,05)$; mientras que los meses de febrero, junio y julio se relacionan de manera negativa (Figura 3a).

De manera similar para el sitio de San Cristóbal, Hidalgo, no se definió un periodo de lluvia estacional que influya de manera significativa en el crecimiento de la especie, únicamente el mes de diciembre del año anterior fue significativo; los meses posteriores correspondientes al año de crecimiento no mostraron una respuesta significativa (Figura 3b).
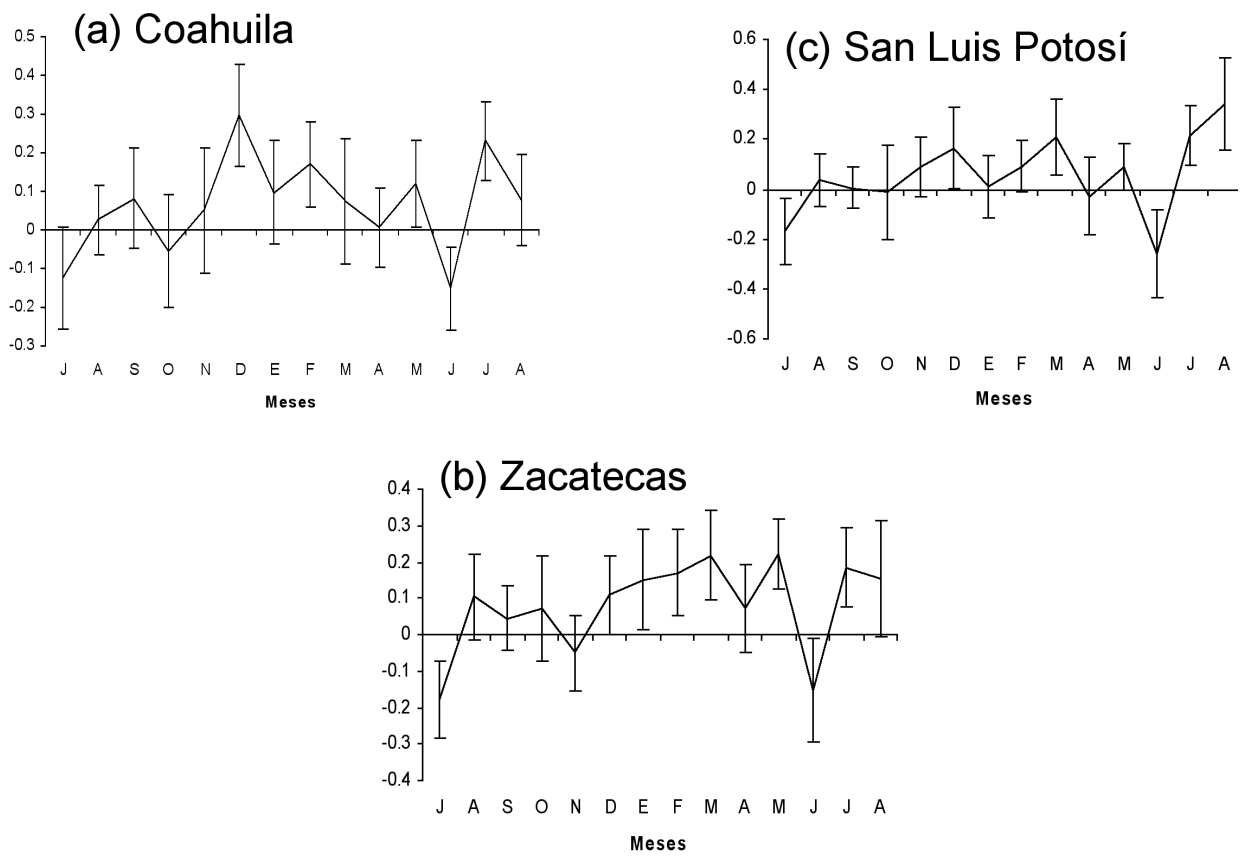

Figura 2. Función de respuesta para poblaciones de Pinus pinceana Gordon en tres estados del norte de México. En cada uno de los estados se comparó la cronología regional (dos poblaciones), dado que presentan una misma variabilidad, con los datos regionales de precipitación. 
(a) Querétaro

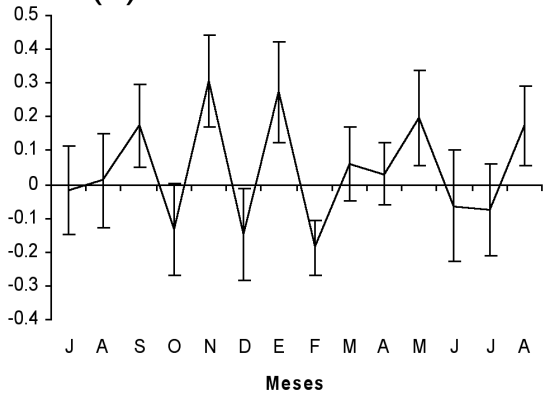

(b) Hidalgo

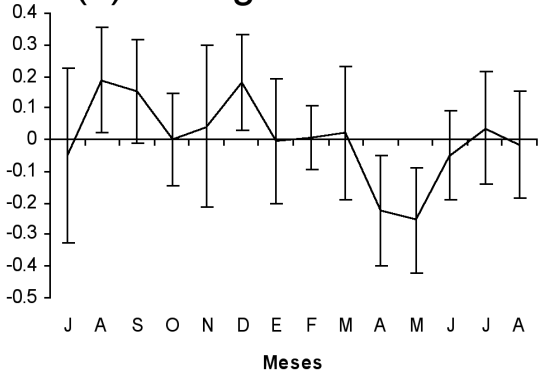

Figura 3. Función de respuesta para dos poblaciones de Pinus pinceana Gordon en el centro de México. Se comparó la cronología regional del estado de Querétaro y la cronología de San Cristóbal del estado de Hidalgo con datos de la estación climática

Peñamiller. (a) Indica que no existe un periodo de precipitación que defina el crecimiento de la especie; sólo los meses de enero, mayo y agosto son significativos $(p<0,05)$. (b) Indica que sólo el mes de diciembre del año anterior tiene una relación significativa $(p<0,05)$ con el crecimiento de la especie. El periodo enero-agosto no presenta una correlación significativa $(p>0,05)$.

\section{DISCUSIÓN}

La información estadística obtenida de las poblaciones de la región centro-norte (MAT, TRI, CAN y CAR) de México indica un alto potencial de la especie para estudios dendrocronológicos; lo anterior fundamentado en la alta calidad de los parámetros estadísticos que reflejan claramente la influencia de las condiciones climáticas y se expresan en la variabilidad en el grosor de sus anillos de crecimiento.

La información obtenida de árboles de Pinus pinceana de los sitios del noreste y centro-norte presenta un comportamiento similar a la de piñoneros y otras coníferas del norte de México que actualmente son empleadas en estudios dendrocronológicos; tal como lo demuestran los resultados de los parámetros estadísticos para Pinus cembroides y Pseudotsuga menziesii (Mirb.) Franco (Cerano, 2004; González-Elizondo et al., 2005; Constante, 2007; Villanueva et al., 2007).
Los valores de correlación obtenidos para la población PAL $(0,77)$, son iguales a los determinados por Cerano (2004) con Pseudotsuga menziesii (Mirb.) Franco para los sitios La Viga, El Coahuilón y Los Pilares en Sierra de Arteaga, Coahuila.

Valores de correlación de $(0,74$ a 0,61 ) en las poblaciones restantes de Pinus pinceana de la región centro-norte y noreste son similares a lo encontrado por Constante (2007) en Pinus cembroides $(0,72)$ en el Ejido Cuauhtémoc, Saltillo, Coahuila y Villanueva et al. (2005) con Pseudotsuga menziesii $(0,73)$ en Bisaloachi, Chihuahua, cuyas series permitieron reconstruir la variabilidad climática.

Los resultados obtenidos de sensibilidad media principalmente para las poblaciones MAT y TRI $(0,69$ y 0,64$)$ fueron superiores a los calculados por Cerano (2004) para El Tarillal $(0,47)$ y al de González-Elizondo et al. (2005) para el 
sitio Cerro Banderas, Durango $(0,38)$ con Pseudotsuga menziesii. En función de estos resultados los valores de sensibilidad media se consideran óptimos con fines de reconstrucciones de la variabilidad climática regional.

En general los valores de desviación estándar en los sitios del noreste NOR $(0,32)$ y centro-norte en TRI $(0,45)$ son similares a los del Coahuilón $(0,32)$, El Tarillal $(0,45)$ (Cerano, 2004) y Cuauhtémoc $(0,30)$ (Constante, 2007).

La autocorrelación de primer orden no mostró gran diferencia entre sitios, los valores extremos en $\operatorname{NOR}(0,21)$ y CAN $(0,29)$ indican que este parámetro se encuentra dentro del límite aceptable con fines dendrocronológicos, al compararlos con valores de este parámetro para la cronología de $P$. menziesii en sitios como La Viga $(0,46)$ y Bisaloachi $(0,19)$ (Cerano, 2004; Villanueva et al., 2007).

Los resultados de la relación señalruido son similares entre los sitios del noreste y centro-norte $(34,63$ a 28,90$)$ considerados altos en el intervalo de valores reportados para El Coahuilón $(0,52)$ y El Tarillal $(23,88)$. No obstante que NOR presentó el valor más bajo $(14,69)$, un valor inferior se reportó para El Tarillal $(11,93)$ y Cerro Barajas $(10,8)$ y sin embargo, tiene potencial con fines dendrocronológicos (Cerano, 2004; González-Elizondo et al., 2005).

El análisis de función de respuesta para Pinus pinceana mostró que los crecimientos de la especie en estas áreas responden de manera significativa a la precipitación estacional de enerojulio. Esto se atribuye a que en el norte de México el crecimiento de las coníferas se encuentra influenciado por la precipitación del periodo frío del año, al ser las lluvias de este periodo de baja intensidad, no logran sobrepasar la capacidad de infiltración, por lo que gran parte de ella se almacena en el suelo y puede ser aprovechada durante el inicio de la estación de crecimiento (Villanueva, 2006).

Esto queda corroborado a través de los estudios dendrocronológicos generados para diversas coníferas en el norte de México, donde se demuestra que especies como Pinus cembroides y Pseudotsuga menziesii responden de manera significativa a la precipitación inviernoprimavera (Stahle et al., 2000; Pohl et al., 2003; Cerano, 2004; González-Elizondo et al., 2005; Constante, 2007).

Los valores obtenidos de los parámetros considerados para el análisis del potencial dendrocronológico de la especie, indicaron que las poblaciones de la región centro MAG, TEP y CRI son sensibles al clima, derivado de los valores de correlación $(0,69)$, sensibilidad media $(0,54)$, desviación estándar $(0,43)$ y autocorrelación de primer orden $(0,24)$; no obstante que sus valores de relación señal-ruido se encontraron por debajo de los observados en los sitios localizados al norte. De acuerdo con González-Elizondo et al. (2005) y Fritts (1976), este parámetro indica que el crecimiento de la especie se encuentra influenciado además por otro tipo de factores ambientales o antropogénicos que están afectando a los árboles de manera individual, a lo que se atribuye el que no se haya determinado un periodo de precipitación anual que influya de manera significativa en el crecimiento de la especie para las poblaciones del centro. A excepción de la población CRI que presenta un valor de señal-ruido más alto, señalando mayor potencial dendroclimático y que con un análisis más detallado de información climática pudiera analizarse el periodo de precipitación que determina el crecimiento de la especie en esta población. 


\section{CONCLUSIONES}

La especie Pinus pinceana mostró alto potencial para estudios dendroclimatológicos, particularmente las poblaciones de la región centro-norte y noreste de México. Este potencial se fundamenta en valores altos de correlación, sensibilidad media, desviación estándar, relación señal-ruido y baja autocorrelación de primer orden.

El crecimiento de Pinus pinceana (región noreste y centro-norte) al igual que otras coníferas del norte de México responde de manera significativa al periodo de precipitación estacional invierno-primavera.

El crecimiento de las poblaciones de la región centro (MAG y CRI) se encuentra influenciado en cierto grado por factores climáticos como lo demostró su alta correlación, sensibilidad y desviación estándar; sin embargo, la baja relación señal-ruido es atribuible al bajo número de muestras analizadas, es recomendable aumentar el tamaño de muestra y observar el comportamiento de esta variable.

El estudio dendroclimático de coníferas sensibles al clima, especialmente Pinus pinceana distribuido en la zona semiárida del noreste de México, constituye una alternativa para extender en el tiempo la información climática, particularmente si consideramos la falta de datos instrumentales, lo que limita un análisis de la disponibilidad de los recursos hídricos y su variabilidad en el tiempo en estas regiones con fines de manejo. La presencia de árboles de Pinus pinceana con edades superiores a los 200 años, sensibles climáticamente y al existir la posibilidad de extender en el tiempo las cronologías, pudiera contribuir a fundamentar programas abocados a su conservación.

\section{AGRADECIMIENTOS}

A la Universidad Autónoma Agraria Antonio Narro por la beca otorgada para los estudios de maestría en ciencias forestales. Al fondo sectorial SemarnatConacyt-2002-Co1-1429 por el apoyo en el trabajo de la etapa de campo. Al personal del laboratorio de dendrocronología del INIFAP CENID-RASPA; ingeniero Julián Cerano Paredes, ingeniero Roque Morán y especialmente a la ingeniera Vicenta Constante García. Finalmente, a los ingenieros Armando Gómez López y Sinhué Ortega Jiménez por su apoyo en laboratorio.

\section{REFERENCIAS}

Cerano, P.J. 2004. Reconstrucción de 350 años de precipitación invierno-primavera para Saltillo, Coahuila. Tesis profesional. Departamento Forestal. Universidad Autónoma Agraria Antonio Narro. Saltillo, Coahuila. 152 p.

Constante, G.V. 2007. Reconstrucción de la precipitación invierno-primavera para los últimos cuatro siglos en el Ejido Cuauhtémoc, Saltillo, Coahuila. Tesis profesional. Departamento Forestal. Universidad Autónoma Agraria Antonio Narro. Saltillo, Coahuila. 72 p.

Cook, E.R. 1987. The decomposition of treering series for environmental studies. Tree-Ring Bulletin 47:37-59.

Fritts, H.C. 1976. Tree-rings and climate. Academic Press. Nueva York. pp. 254260.

Fritts, H.C. 1990. Statistical reconstruction of spatial variations in climate. In, Cook E., Kairiukstis L. (eds.), Methods of dendrochronology: applications in the environmental science. Kluwer Academic Publishers, Dordrecht, pp. 193-210 
González-Elizondo, M., E. Jurado, J. Návar, M.S. González-Elizondo, J. Villanueva-Díaz, O. Aguirre. y J. Jiménez. 2005. Tree-rings and climate relationships for Douglas-fir chronologies from the Sierra Madre Occidental, Mexico: a 1681-2001 rain reconstruction. Forest Ecology and Management (213):39-53.

Grissino-Mayer, H.D. 2001. Evaluating crossdating, accuracy: a manual and tutorial for the computer program COFECHA. Tree-Ring Research 57(2):205-221.

Holmes, R.L. 1983. Computer-assisted quality control in tree-ring dating and measurement. Tree-Ring Bulletin 43:69-78.

Pohl, K., M.D. Therrell, J.S. Blay, N. Ayotte, J.J. Cabrera, S.C. Díaz, E.O. Cornejo, J.A. Elvir, M.E. Gonzalez, D. Opland, J. Park, G. Pederson, S.S. Bernal, L.S. Vazquez, J. Villanueva-Díaz y D.W. Stahle. 2003. A cool season precipitation reconstruction for Saltillo, Mexico. Tree-Ring Research 59(1):11-19.

Semarnat. 2004. NOM-ECOL-050-2001. http://www.sma.df.gob.mx/sma/dow nload/archivos/sedesol_nom_059_e col_2004.pdf

Stat Soft Inc. 2000. Statistica Kernel versión 5,5 A. 1984-2000.

Stahle, D.W., J. Villanueva-Díaz, M.K. Cleveland, M.D. Therrell, G.J. Paull, B.T. Burns, W. Salinas, H. Suzán y P.Z. Fule. 2000. Recent tree-ring research in Mexico. In F.A. Roig (comp.), Dendrocronología en América Latina. EDIUNC. Argentina. pp. 285-306.

Stokes M.A. y T.L. Smiley. 1968. An introduction to the tree-ring dating. The University of Arizona Press. 73 p.

Villanueva, D.J., D.W. Stahle, M.K. Cleaveland y M.D. Therrell. 2000. Estado actual de la dendrocronología en México. Ciencia Forestal 25(88):536.

Villanueva, D.J., J. Cerano-P., D.W. Stahle, M.D. Therrell, M.K. Cleaveland e I.C. Sánchez. 2004. Elementos básicos de la dendrocronología y sus aplicaciones en México. Folleto técnico No. 2. INIFAP CENID-RASPA. Gómez Palacio, Dgo. $37 \mathrm{p}$.

Villanueva, D.J., J. Cerano-P., D.W. Sthale, M.D. Therrel, M.K. Cleaveland y B.H. Luckman. 2005. Estudios paleoclimáticos en México utilizando anillos de crecimiento de especies arbóreas. Capítulo I. Libro técnico No. 1. Contribución al estudio de los servicios ambientales. Sagarpa, INIFAP. pp. 7-32.

Villanueva, D.J., D.W. Stahle, B.H. Luckman, J. Cerano-P., M.D. Therrell, R. Morán, M.L. Cleaveland. 2007. Potencial dendrocronológico de Taxodium mucronatum Ten. y acciones para su conservación en México. Ciencia Forestal 32(101):9-37.

Manuscrito recibido el 21 de abril de 2008

Aceptado el 6 de abril de 2009

Este documento se debe citar como: Santillán-Hernández, M., E.H. Cornejo-Oviedo, J. Villanueva-Díaz, J. CeranoParedes, S. Valencia-Manzo y M.A. Capó-Arteaga. 2010. Potencial dendroclimático de Pinus pinceana Gordon en la Sierra Madre Oriental. Madera y Bosques 16(1):17-29. 